\title{
QUANTILE AUTOREGRESSION
}

\author{
ROGER KOENKER AND ZHIJIE XIAO
}

\begin{abstract}
We consider quantile autoregression (QAR) models in which the autoregressive coefficients can be expressed as monotone functions of a single, scalar random variable. The models can capture systematic influences of conditioning variables on the location, scale and shape of the conditional distribution of the response, and therefore constitute a significant extension of classical constant coefficient linear time series models in which the effect of conditioning is confined to a location shift. The models may be interpreted as a special case of the general random coefficient autoregression model with strongly dependent coefficients. Statistical properties of the proposed model and associated estimators are studied. The limiting distributions of the autoregression quantile process are derived. Quantile autoregression inference methods are also investigated. Empirical applications of the model to the U.S. unemployment rate and U.S. gasoline prices highlight the potential of the model.
\end{abstract}

\section{INTRODUCTION}

Constant coefficient linear time series models have played an enormously successful role in econometrics, and gradually various forms of random coefficient time series models have also emerged as viable competitors in particular fields of application. One variant of the latter class of models, although perhaps not immediately recognizable as such, is the linear quantile autoregression model. This model has received considerable attention in the theoretical literature, and can be easily estimated with the quantile regression methods proposed in Koenker and Bassett (1978). Curiously, however, all of the theoretical work dealing with this model (that we are aware of) focuses exclusively on the iid innovation case that restricts the autoregressive coefficients to be independent of the specified quantiles. In this paper we seek to relax this restriction and consider linear quantile autoregression models whose autoregressive (slope) parameters may vary with quantiles $\tau \in[0,1]$. We hope that these models might expand the modeling options for economic time series that display asymmetric dynamics or local persistency.

Key words and phrases. random coefficient model, asymmetric dynamics.

JEL Classification: C14, C22. Corresponding author: Roger Koenker, Department of Economics, University of Illinois, Champaign, Il, 61820. Email: rkoenker@uiuc.edu.

Version March 4, 2004. This research was partially supported by NSF grant SES-02-40781. The authors would like to thank Steve Portnoy and Peter Phillips for valuable discussions regarding this work. 
In recent years, considerable research effort has been devoted to modifications of traditional unit root models to incorporate the effect of various types of shocks. An important motivation for such modifications is the introduction of asymmetries into economic dynamics. It is widely acknowledged that many important economic variables may display asymmetric adjustment paths (e.g. Neftci (1984), Enders and Granger (1998)). The observation that firms are more apt to increase than to reduction in prices is a key feature of many macroeconomic models. Beaudry and Koop (1993) showed that positive shocks to U.S. GDP are more persistent than negative shocks, indicating asymmetric business cycle dynamics over different quantiles of the innovation process. In addition, while it is recognized that output fluctuations are persistent, less persistent results are also found at longer horizons (Beaudry and Koop (1993)), indicating the existence of "local persistency" or "temporal persistency" in economic time series. See, inter alia, Delong and Summers (1986), Hamilton (1989), Sichel (1989), Diebold and Rudebusch (1990), Evans and Wachtel (1993), Potter (1995), Bradley and Jansen (1997), Hess and Iwata (1997), and Kuan and Huang (2001)) among others on the study of asymmetric dynamics in economic time series. A related development is the growing literature on threshold autoregression (TAR) with unit roots (e.g. Balke and Fomby (1997); Tsay (1997); Gonzalez and Gonzalo (1998); Hansen (1999); and Caner and Hansen (2001)). In particular, Tsay (1997) proposed a unit root test when the innovations follow a threshold process; Gonzalez and Gonzalo (1998) studied a TAR(1) model that allows for a unit root; Caner and Hansen (2001) develops an asymptotic theory of inference for an unrestricted two regime TAR model with a unit root.

We believe that quantile regression methods can provide an alternative way to study asymmetric dynamics and persistency in economic time series. Linton and Whang (2004) have recently proposed related "quantilogram" inference methods for exploring linear dependence in time series at various quantiles. In this paper, we propose a new quantile autoregression (QAR) model whose autoregressive coefficient may take different values (possibly unity) over different quantiles of the innovation process. We show that some forms of the model can exhibit unit-root like tendencies or even temporarily explosive behavior, but with occasional episodes of mean reversion sufficient to insure stationarity. The models lead to interesting new hypotheses and inference apparatus for economic time series.

The paper is organized as follows: We introduce the model and study some basic statistical properties of the QAR process in Section 2. Section 3 develops the limiting distribution of the QAR estimator. Section 4 considers some restrictions imposed on the model by the monotonicity requirement on the conditional quantile functions. Statistical inference, including testing for asymmetric dynamics, is explored in Section 5. Section 6 reports a Monte Carlo experiment on the sampling performance of the proposed inference procedure. Empirical applications to the U.S. unemployment rate 
and the U.S. price of gasoline are discussed in Section 7. Proofs are provided in the Appendix.

\section{The Model}

There is a substantial theoretical literature, including Weiss (1987), Knight (1989), Koul and Saleh(1995), Koul and Mukherjee(1994), Hercé (1996), Hasan and Koenker (1997), Jurečková and Hallin (1999) dealing with the linear quantile autoregression model. In this model the $\tau$-th conditional quantile function of the response $y_{t}$ is expressed as a linear function of lagged values of the response. But a striking feature of this literature is that it has focused exclusively on the case of iid innovations in which the conditioning variables play their classical role of shifting the location of the conditional density of $y_{t}$, but they have no effect on conditional scale or shape. In this paper we wish to study estimation and inference in a more general class of quantile autoregressive (QAR) models in which all of the autoregressive coefficients are allowed to be $\tau$-dependent, and therefore are capable of altering the location, scale and shape of the conditional densities.

2.1. The Model. Let $\left\{U_{t}\right\}$ be a sequence of iid standard uniform random variables, and consider the $p$ th order autoregressive process,

$$
y_{t}=\theta_{0}\left(U_{t}\right)+\theta_{1}\left(U_{t}\right) y_{t-1}+\cdots+\theta_{p}\left(U_{t}\right) y_{t-p},
$$

where the $\theta_{j}$ 's are unknown functions $[0,1] \rightarrow \mathbb{R}$ that we will want to estimate. Provided that the right hand side of (1) is monotone increasing in $U_{t}$, it follows that the $\tau$ th conditional quantile function of $y_{t}$ can be written as,

$$
Q_{y_{t}}\left(\tau \mid y_{t-1}, \ldots, y_{t-p}\right)=\theta_{0}(\tau)+\theta_{1}(\tau) y_{t-1}+\ldots+\theta_{p}(\tau) y_{t-p}
$$

or somewhat more compactly as,

$$
Q_{y_{t}}\left(\tau \mid \mathcal{F}_{t-1}\right)=x_{t}^{\top} \theta(\tau)
$$

where $x_{t}=\left(1, y_{t-1}, \ldots, y_{t-p}\right)^{\top}$, and $\mathcal{F}_{t}$ is the $\sigma$-field generated by $\left\{y_{s}, s \leq t\right\}$. The transition from (1) to (2) is an immediate consequence of the fact that for any monotone increasing function $g$ and standard uniform random variable, $U$, we have

$$
Q_{g(U)}(\tau)=g\left(Q_{U}(\tau)\right)=g(\tau),
$$

where $Q_{U}(\tau)=\tau$ is the quantile function of $U$. In the above model, the autoregressive coefficients may be $\tau$-dependent and thus can vary over the quantiles. The conditioning variables not only shift the location of the distribution of $y_{t}$, but also may alter the scale and shape of the conditional distribution. We will refer to this model as the QAR(p) model.

We will argue that QAR models can play a useful role in expanding the modeling territory between classical stationary linear time series models and their unit root alternatives. To illustrate this in the QAR(1) case, consider the model

$$
Q_{y_{t}}\left(\tau \mid \mathcal{F}_{t-1}\right)=\theta_{0}(\tau)+\theta_{1}(\tau) y_{t-1}
$$


with $\theta_{0}(\tau)=\sigma \Phi^{-1}(\tau)$, and $\theta_{1}(\tau)=\min \left\{\gamma_{0}+\gamma_{1} \tau, 1\right\}$ for $\gamma_{0} \in(0,1)$ and $\gamma_{1}>0$. In this model if $U_{t}>\left(1-\gamma_{0}\right) / \gamma_{1}$ the model generates the $y_{t}$ according to the standard Gaussian unit root model, but for smaller realizations of $u_{t}$ we have a mean reversion tendency. Thus, the model exhibits a form of asymmetric persistence in the sense that sequences of strongly positive innovations tend to reinforce its unit root like behavior, while occasional negative realizations induce mean reversion and thus undermine the persistence of the process. The classical Gaussian AR(1) model is obtained by setting $\theta_{1}(\tau)$ to a constant.

The formulation in (4) reveals that the model may be interpreted as rather unusual form of random coefficient autoregressive (RCAR) model. Such models arise naturally in many time series applications. Discussions of the role of RCAR models can be found in, inter alia, Nicholls and Quinn (1982), Tjøstheim(1986), Pourahmadi (1986), Brandt (1986), Karlsen(1990), and Tong (1990). In contrast to most of the literature on RCAR models, in which the coefficients are typically assumed to be stochastically independent of one another, the QAR model has coefficients that are functionally dependent.

Monotonicity of the conditional quantile functions imposes some discipline on the forms taken by the $\theta$ functions. This discipline essentially requires that the function $Q_{y_{t}}\left(\tau \mid y_{t-1}, \ldots, y_{t-p}\right)$ is monotone in $\tau$ in some relevant region $\Upsilon$ of $\left(y_{t-1}, \ldots, y_{t-p}\right)$-space. The correspondance between the random coefficient formulation of the QAR model (1) and the conditional quantile function formulation (2) presupposes the monotonicity of the latter in $\tau$. In the region $\Upsilon$ where this monotonicity holds (1) can be regarded as a valid mechanism for simulating from the QAR model (2). Of course, model (1) can, even in the absence of this monotonicity, be taken as a valid data generating mechanism, however the link to the strictly linear conditional quantile model is no longer valid. At points where the monotonicity is violated the conditional quantile functions corresponding to the model described by (1) have linear "kinks". Attempting to fit such piecewise linear models with linear specifications can be hazardous. We will return to this issue in the discussion of Section 4. In the next section we briefly describe some essential features of the QAR model.

2.2. Properties of the QAR Process. The QAR $(\mathrm{p})$ model (1) can be reformulated in more conventional random coefficient notation as,

$$
y_{t}=\mu_{0}+\alpha_{1, t} y_{t-1}+\cdots+\alpha_{p, t} y_{t-p}+u_{t}
$$

where $\mu=E \theta_{0}\left(U_{t}\right), u_{t}=\theta_{0}\left(U_{t}\right)-\mu$, and $\alpha_{j, t}=\theta_{j}\left(U_{t}\right)$, for $j=1, \ldots, p$. Thus, $\left\{u_{t}\right\}$ is an iid sequence of random variables with distribution function $F(\cdot)=\theta_{0}^{-1}(\cdot+\mu)$, and the $\alpha_{j, t}$ coefficients are functions of this $u_{t}$ innovation random variable. The QAR $(p)$ process (5) can be expressed as an $p$-dimensional vector autoregression process of order 1:

$$
Y_{t}=\Gamma+A_{t} Y_{t-1}+V_{t}
$$


with

$$
\Gamma=\left[\begin{array}{c}
\mu_{0} \\
0_{p-1}
\end{array}\right], A_{t}=\left[\begin{array}{cc}
A_{p-1, t} & \alpha_{p, t} \\
I_{p-1} & 0_{p-1}
\end{array}\right], V_{t}=\left[\begin{array}{c}
u_{t} \\
0_{p-1}
\end{array}\right]
$$

where $A_{p-1, t}=\left[\begin{array}{lll}\alpha_{1, t}, & \ldots, & \alpha_{p-1, t}\end{array}\right], Y_{t}=\left[y_{t}, \cdots, y_{t-p+1}\right]^{\top}$, and $0_{p-1}$ is the $(p-1)$ dimensional vector of zeros. In the Appendix, we show that under regularity conditions given in the following Theorem, an $\mathcal{F}_{t}$-measurable solution for (5) can be found.

To formalize the foregoing discussion and facilitate later asymptotic analysis, we introduce the following conditions.

A.1: $\left\{u_{t}\right\}$ are iid random variables with mean 0 and variance $\sigma^{2}<\infty$. The distribution function of $u_{t}, F$, has a continuous density $f$ with $f(u)>0$ on $\mathcal{U}=\{u: 0<F(u)<1\}$.

A.2: Let $E\left(A_{t} \otimes A_{t}\right)=\Omega_{A}$, the eigenvalues of $\Omega_{A}$ have moduli less than unity.

A.3: Denote the conditional distribution function $\operatorname{Pr}\left[y_{t}<\cdot \mid \mathcal{F}_{t-1}\right]$ as $F_{t-1}(\cdot)$ and its derivative as $f_{t-1}(\cdot), f_{t-1}$ is uniformly integrable on $\mathcal{U}$.

Theorem 2.1. Under assumptions A.1 and A.2, the time series $y_{t}$ given by (5) is covariance stationary and satisfies a central limit theorem

$$
\frac{1}{\sqrt{n}} \sum_{t=1}^{n} y_{t} \Rightarrow N\left(\mu_{y}, \omega_{y}^{2}\right)
$$

where

$$
\mu_{y}=\frac{\mu_{0}}{1-\sum_{j=1}^{p} \mu_{p}} ; \omega_{y}^{2}=\lim \frac{1}{n} E\left[\sum_{t=1}^{n}\left(y_{t}-\mu_{y}\right)\right]^{2}, \text { and } \mu_{j}=E\left(\alpha_{j, t}\right), j=1, \ldots, p .
$$

To illustrate some important features of the QAR process, we consider the simplest case of QAR(1) process,

$$
y_{t}=\alpha_{t} y_{t-1}+u_{t}
$$

where $\alpha_{t}=\theta_{1}\left(U_{t}\right)$ and $u_{t}=\theta_{0}\left(U_{t}\right)$ corresponding to (4), whose properties are summarized in the following corollary.

Corollary 2.1. If $y_{t}$ is determined by (6), and $\omega_{\alpha}^{2}=E\left(\alpha_{t}\right)^{2}<1$, under assumption A.1, $y_{t}$ is covariance stationary and satisfies a central limit theorem

$$
\frac{1}{\sqrt{n}} \sum_{t=1}^{n} y_{t} \Rightarrow N\left(0, \omega_{y}^{2}\right)
$$

where

$$
\omega_{y}^{2}=\frac{1+\mu_{\alpha}}{\left(1-\mu_{\alpha}\right)\left(1-\omega_{\alpha}^{2}\right)} \sigma^{2}
$$

with $\mu_{\alpha}=E\left(\alpha_{t}\right)<1$. 
In the example given in Section 2.1, $\alpha_{t}=\theta_{1}\left(U_{t}\right)=\min \left\{\gamma_{0}+\gamma_{1} U_{t}, 1\right\} \leq 1$, and $\operatorname{Pr}\left(\left|\alpha_{t}\right|<1\right)>0$, the condition of Corollary 2 holds and the process $y_{t}$ is globally stationary but can still display local (and asymmetric) persistency in the presence of certain type of shocks (positive shocks in the example). Corollary 2 also indicates that even with $\alpha_{t}>1$ over some range of quantiles, as long as $\omega_{\alpha}^{2}=E\left(\alpha_{t}\right)^{2}<1$, $y_{t}$ can still be covariance stationary in the long run. Thus, a quantile autoregressive process may allow for some (transient) forms of explosive behavior while maintaining stationarity in the long run.

Under the assumptions in Corollary 2, by recursively substituting in (6), we can see that

$$
y_{t}=\sum_{j=0}^{\infty} \beta_{t, j} u_{t-j}, \text { where } \beta_{t, 0}=1, \text { and } \beta_{t, j}=\prod_{i=0}^{j-1} \alpha_{t-i}, \text { for } j \geq 1 .
$$

is a stationary $\mathcal{F}_{t}$-measurable solution to (6). In addition, if $\sum_{j=0}^{\infty} \beta_{t, j} v_{t-j}$ converges in $L^{p}$, then $y_{t}$ has a finite $p$-th order moment. The $\mathcal{F}_{t}$-measurable solution of (6) gives a doubly stochastic $M A(\infty)$ representation of $y_{t}$. In particular, the impulse response of $y_{t}$ to a shock $u_{t-j}$ is stochastic and is given by $\beta_{t, j}$. On the other hand, although the impulse response of the quantile autoregressive process is stochastic, it does converge (to zero) in mean square (and thus in probability) as $j \rightarrow \infty$, corroborating the stationarity of $y_{t}$. If we denote the autocovariance function of $y_{t}$ by $\gamma_{y}(h)$, it is easy to verify that

$$
\gamma_{y}(h)=\mu_{\alpha}^{|h|} \sigma_{y}^{2}, \text { where } \sigma_{y}^{2}=\frac{\sigma^{2}}{1-\omega_{\alpha}^{2}} .
$$

Remark 2.1. Comparing to the $Q A R(1)$ process $y_{t}$, if we consider a conventional $A R(1)$ process with autoregressive coefficient $\mu_{\alpha}$ and denote the corresponding process by $\underline{y}_{t}$, the long-run variance of $y_{t}$ (given by $\omega_{y}^{2}$ ) is (as expected) larger than that of $\underline{y}_{t}$. The additional variance the $Q A R$ process $y_{t}$ comes from the variation of $\alpha_{t}$. In fact, $\omega_{y}^{2}$ can be decomposed into the summation of the long-run variance of $\underline{y}_{t}$ and an additional term that is determined by the variance of $\alpha_{t}$ :

$$
\omega_{y}^{2}=\underline{\omega}_{y}^{2}+\frac{\sigma^{2}}{\left(1-\mu_{\alpha}\right)^{2}\left(1-\omega_{\alpha}^{2}\right)} \operatorname{Var}\left(\alpha_{t}\right),
$$

where $\underline{\omega}_{y}^{2}=\sigma^{2} /\left(1-\mu_{\alpha}\right)^{2}$ is the long-run variance of $\underline{y}_{t}$.

We consider estimation and related inference on the QAR model in the next two sections.

\section{Estimation}

Estimation of the quantile autoregressive model (3) involves solving the problem

$$
\min _{\theta \in \mathbb{R}^{p+1}} \sum_{t=1}^{n} \rho_{\tau}\left(y_{t}-x_{t}^{\top} \theta\right)
$$


where $\rho_{\tau}(u)=u(\tau-I(u<0))$ as in Koenker and Bassett (1978). Solutions, $\widehat{\theta}(\tau)$, are called autoregression quantiles. Given $\widehat{\theta}(\tau)$, the $\tau$-th conditional quantile function of $y_{t}$, conditional on $x_{t}$, could be estimated by,

$$
\hat{Q}_{y_{t}}\left(\tau \mid x_{t}\right)=x_{t}^{\top} \hat{\theta}(\tau)
$$

and the conditional density of $y_{t}$ can be estimated by the difference quotients,

$$
\hat{f}_{y_{t}}\left(\tau \mid x_{t-1}\right)=\left(\tau_{i}-\tau_{i-1}\right) /\left(\hat{Q}_{y_{t}}\left(\tau_{i} \mid x_{t-1}\right)-\hat{Q}_{y_{t}}\left(\tau_{i-1} \mid x_{t-1}\right)\right),
$$

for some appropriately chosen sequence of $\tau$ 's.

If we denote $E\left(y_{t}\right)$ as $\mu_{y}, E\left(y_{t} y_{t-j}\right)$ as $\gamma_{j}$, and let $\Omega_{0}=E\left(x_{t} x_{t}^{\top}\right)=\lim n^{-1} \sum_{t=1}^{n} x_{t} x_{t}^{\top}$, then

$$
\Omega_{0}=\left[\begin{array}{cc}
1 & \mu_{y} \\
\mu_{y} & \Omega_{y}
\end{array}\right]
$$

where

$$
\Omega_{y}=\left[\begin{array}{ccc}
\gamma_{0} & \cdots & \gamma_{p-1} \\
\vdots & \ddots & \vdots \\
\gamma_{p-1} & \cdots & \gamma_{0}
\end{array}\right]
$$

In the special case of $\mathrm{QAR}(1)$ model $(6), \Omega_{0}=E\left(x_{t} x_{t}^{\top}\right)=\operatorname{diag}\left[1, \gamma_{0}\right], \gamma_{0}=E\left[y_{t}^{2}\right]$. Let $\Omega_{1}=\lim n^{-1} \sum_{t=1}^{n} f_{t-1}\left[F_{t-1}^{-1}(\tau)\right] x_{t} x_{t}^{\top}$, and define $\Sigma=\Omega_{1}^{-1} \Omega_{0} \Omega_{1}^{-1}$. The asymptotic distribution of $\widehat{\theta}(\tau)$ is summarized in the following Theorem.

Theorem 3.1. Under assumptions A.1 - A.3,

$$
\Sigma^{-1 / 2} \sqrt{n}(\widehat{\theta}(\tau)-\theta(\tau)) \Rightarrow B_{k}(\tau)
$$

where $B_{k}(\tau)$ represents a $k$-dimensional standard Brownian Bridge, $k=p+1$.

By definition, for any fixed $\tau, B_{k}(\tau)$ is $\mathcal{N}\left(0, \tau(1-\tau) I_{k}\right)$. In the important special case with constant coefficients, $\Omega_{1}=f\left[F^{-1}(\tau)\right] \Omega_{0}$, where $f(\cdot)$ and $F(\cdot)$ are the density and distribution functions of $u_{t}$, respectively. We state this result in the following corollary.

Corollary 3.1. Under assumptions A.1 - A.3, if the coefficients $\alpha_{j t}$ are constants, then

$$
\left[f\left[F^{-1}(\tau)\right]^{-1} \Omega_{0}\right]^{1 / 2} \sqrt{n}(\widehat{\theta}(\tau)-\theta(\tau)) \Rightarrow B_{k}(\tau) .
$$

An alternative form of the model that is widely used in economic applications is

$$
y_{t}=\mu_{0}+\delta_{0, t} y_{t-1}+\sum_{j=1}^{p-1} \delta_{j, t} \Delta y_{t-j}+u_{t},
$$

where, corresponding to (5),

$$
\delta_{0, t}=\sum_{s=1}^{p} \alpha_{s, t}, \delta_{j, t}=-\sum_{s=j+1}^{p} \alpha_{s, t}, j=1, \cdots, p-1 .
$$


In the above transformed model, $\delta_{0, t}$ is the critical parameter corresponding the largest autoregressive root. Let $z_{t}=\left(1, y_{t-1}, \Delta y_{t-1}, \ldots, \Delta y_{t-p+1}\right)^{\top}$, we may write the quantile regression counterpart of (9) as

$$
Q_{y_{t}}\left(\tau \mid \mathcal{F}_{t-1}\right)=z_{t}^{\top} \delta(\tau)
$$

where

$$
\delta(\tau)=\left(\alpha_{0}(\tau), \delta_{0}(\tau), \delta_{1}(\tau), \cdots, \delta_{p-1}(\tau)\right)^{\top} .
$$

The limiting distributions of the quantile regression estimators $\widehat{\delta}(\tau)$ can be obtained from our previous analysis. If we define

$$
J=\left[\begin{array}{ccccc}
1 & 0 & 0 & \cdots & 0 \\
0 & 1 & 1 & \cdots & 1 \\
0 & 0 & -1 & & -1 \\
& & & \ddots & \\
0 & 0 & 0 & \cdots & -1
\end{array}\right], \text { and } \Delta=J \Sigma J
$$

then we have, under assumptions A.1 - A.3,

$$
\Delta^{-1 / 2} \sqrt{n}(\widehat{\delta}(\tau)-\delta(\tau)) \Rightarrow B_{k}(\tau)
$$

If we focus our attention on the largest autoregressive root $\delta_{0, t}$ in the ADF type regression (9) and consider the special case that $\delta_{j, t}=$ constant for $j=1, \ldots, p-1$, then, a result similar to Corollary 2 can be obtained.

Corollary 3.2. Under assumptions A.1-A.3, if $\delta_{j, t}=$ constant for $j=1, \ldots, p-1$, and $\delta_{0, t} \leq 1$ and $\left|\delta_{0, t}\right|<1$ with positive probability, then the time series $y_{t}$ given by (9) is covariance stationary and satisfies a central limit theorem.

\section{Quantile Monotonicity}

As in other linear quantile regression applications, linear QAR models should be cautiously interpreted as useful local approximations to more complex nonlinear global models. If we take the linear form of the model too literally then obviously at some point, or points, there will be "crossings" of the conditional quantile functions - unless these functions are precisely parallel in which case we are back to the pure location shift form of the model. This crossing problem appears more acute in the autoregressive case than in ordinary regression applications since the support of the design space, i.e. the set of $x_{t}$ that occur with positive probability, is determined within the model. Nevertheless, we may still regard the linear models specified above as valid local approximations over a region of interest.

It should be stressed that the estimated conditional quantile functions,

$$
\hat{Q}_{y}(\tau \mid x)=x^{\top} \widehat{\theta}(\tau)
$$




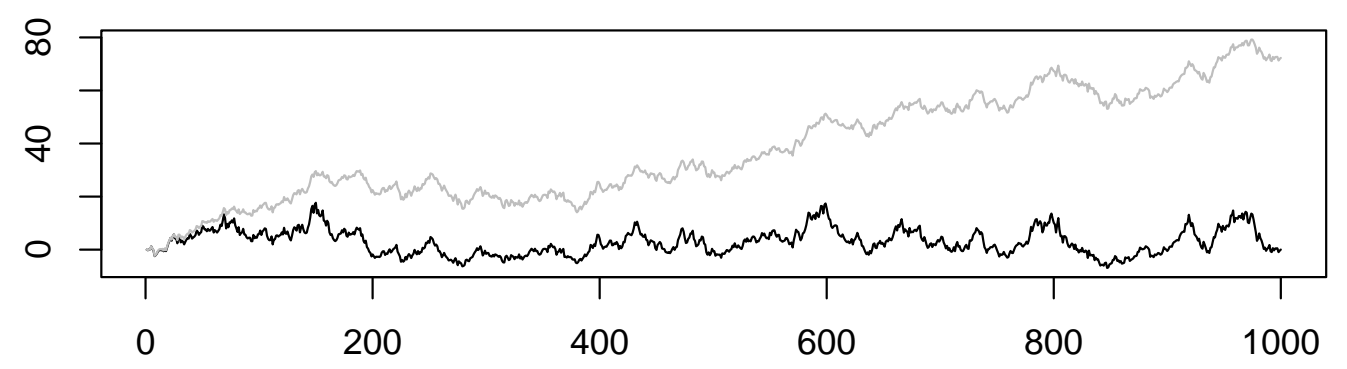

FiguRE 1. QAR and Unit Root Time-Series: The figure contrasts two time series generated by the same sequence of innovations. The grey sample path is a random walk with standard Gaussian innovations; the black sample path illustrates a QAR series generated by the same innovations with random $\mathrm{AR}(1)$ coefficient $.85+.25 \Phi\left(u_{t}\right)$. The latter series although exhibiting explosive behavior in the upper tail is stationary as described in the text.

are guaranteed to be monotone at the mean design point, $x=\bar{x}$, as shown in Bassett and Koenker (1982), for linear quantile regression models. Crossing, when it occurs, is generally confined to outlying regions of the design space. In our random coefficient view of the QAR model,

$$
y_{t}=x_{t}^{\top} \theta\left(U_{t}\right)
$$

we express the observable random variable $y_{t}$ as a linear function conditioning covariates. But rather than assuming that the coordinates of the vector $\theta$ are independent random variables we adopt a diametrically opposite viewpoint - that they are perfectly functionally dependent, all driven by a single random uniform variable. If the functions $\left(\theta_{0}, \ldots, \theta_{p}\right)$ are all monotonically increasing then the coordinates of the random vector $\alpha_{t}$ are said to be comonotonic in the sense of Schmeidler (1986). ${ }^{1}$ This is often the case, but there are important cases for which this monotonicity fails. What then?

What really matters is that we can find a linear reparameterization of the model that does exhibit comonotonicity over some relevant region of covariate space. Since

\footnotetext{
${ }^{1}$ Random variables $X$ and $Y$ on a probability space $(\Omega, \mathcal{A}, P)$ are said to be comonotonic if there are monotone functions, $g$ and $h$ and a random variable $Z$ on $(\Omega, \mathcal{A}, P)$ such that $X=g(Z)$ and $Y=h(Z)$.
} 


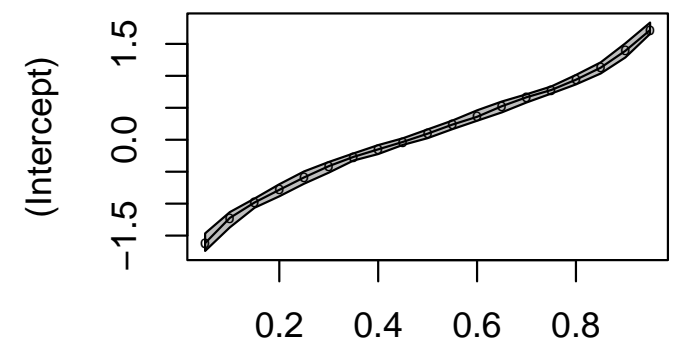

tau

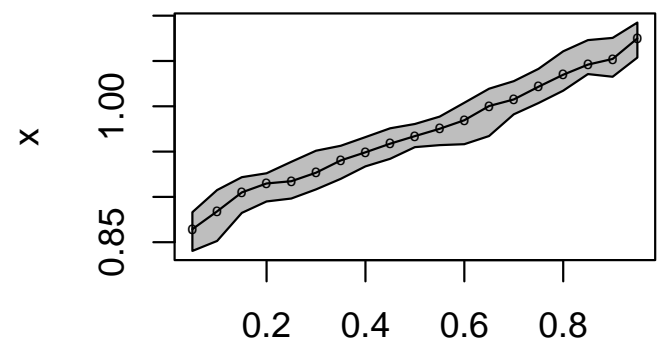

tau

FiguRE 2. Estimating the QAR model: The figure illustrates estimates of the QAR(1) model based on the black time series of the previous figure. The left panel represents the intercept estimate at 19 equally spaced quantiles, the right panel represents the $\mathrm{AR}(1)$ slope estimate at the same quantiles. The shaded region is a .90 confidence band. Note that the slope estimate quite accurate reproduces the linear form of the QAR(1) coefficient used to generate the data.

for any nonsingular matrix $A$ we can write,

$$
Q_{y}(\tau \mid x)=x^{\top} A^{-1} A \theta(\tau)
$$

we can choose $p+1$ linearly independent design points $\left\{x_{s}: s=1, \ldots, p+1\right\}$ where $Q_{y}\left(\tau \mid x_{s}\right)$ is monotone in $\tau$, then choosing the matrix A so that $A x_{s}$ is the sth unit basis vector for $\mathbb{R}^{p+1}$ we have

$$
Q_{y}\left(\tau \mid x_{s}\right)=\gamma_{s}(\tau)
$$

where $\gamma=A \theta$. And now inside the convex hull of of our selected points we have a comonotonic random coefficient representation of the model. In effect, we have simply reparameterized the design so that the $p+1$ coefficients are the conditional quantile functions of $y_{t}$ at the selected points. The fact that quantile functions of sums of nonnegative comonotonic random variables are sums of their marginal quantile functions, see e.g. Denneberg(1994), allows us to interpolate inside the convex hull. Of course, linear extrapolation is also possible but we must be cautious about possible violations of the monotonicity requirement in this region.

The interpretation of linear conditional quantile functions as approximations to the local behavior in central range of the covariate space should always be regarded as provisional; richer data sources can be expected to yield more elaborate nonlinear specifications that would have validity over larger regions. Figure 1 illustrates a 

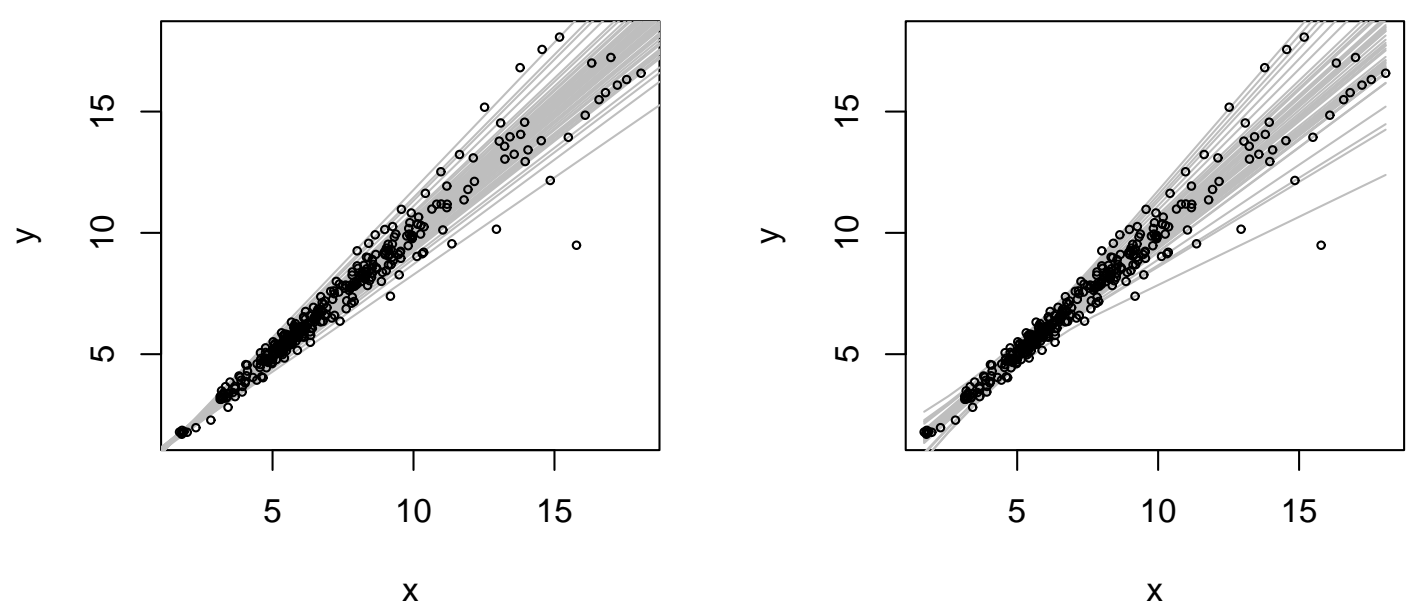

Figure 3. QAR(1) Model of U.S. Short Term Interest Rate: The AR(1) scatterplot of the U.S. three month rate is superimposed in the left panel with 49 equally spaced estimates of linear conditional quantile functions. In the right panel the model is augmented with a nonlinear (quadratic) component. The introduction of the quadratic component alleviates some nonmonotonicity in the estimated quantiles at low interest rates.

realization of the simple QAR(1) model described in Section 2. The black sample path shows 1000 observations generated from the model (4) with $\operatorname{AR}(1)$ coefficient $\theta_{1}(u)=.85+.25 u$ and $\theta_{0}(u)=\Phi^{-1}(u)$. The grey sample path depicts the a random walk generated from the same innovation sequence, i.e. the same $\theta_{0}\left(U_{t}\right)$ 's but with constant $\theta_{1}$ equal to one. It is easy to verify that the QAR(1) form of the model satisfies the stationarity conditions of Section 2.2, and despite the explosive character of its upper tail behavior we observe that the series appears quite stationary, at least by comparison to the random walk series. Estimating the QAR(1) model at 19 equally spaced quantiles yields the intercept and slope estimates depicted in Figure 2.

Figure 3 depicts estimated linear conditional quantile functions for short term (three month) US interest rates using the QAR(1) model superimposed on the AR(1) scatter plot. In this example the scatterplot shows clearly that there is more dispersion at higher interest rates, with nearly degenerate behavior at very low rates. The fitted linear quantile regression lines in the left panel show little evidence of crossing, but at rates below .04 there are some violations of the monotonicity requirement in the fitted quantile functions. Fitting the data using a somewhat more 

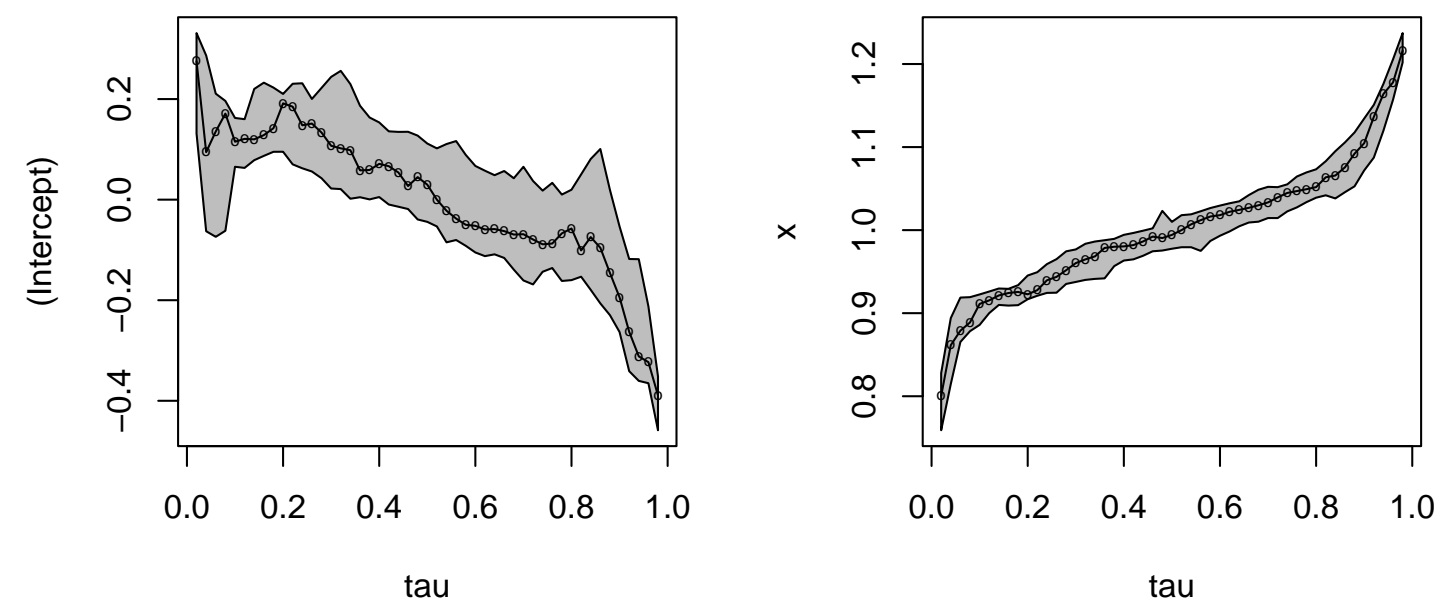

Figure 4. QAR(1) Model of U.S. Short Term Interest Rate: The QAR(1) estimates of the intercept and slope parameters for 19 equally spaced quantile functions are illustrated in the two plots. Note that the slope parameter is, like the prior simulated example, explosive in the upper tail but mean reverting in the lower tail.

complex nonlinear (in variables) model by introducing a another additive component $\theta_{2}(\tau)\left(y_{t-1}-\delta\right)^{2} I\left(y_{t-1}<\delta\right)$ with $\delta=8$ in our example we can eliminate the problem of the crossing of the fitted quantile functions. In Figure 4 depicting the fitted coefficients of the QAR(1) model and their confidence region, we see that the estimated slope coefficient of the QAR(1) model has somewhat similar appearance to the simulated example. Even more flexible models may be needed in other settings. A B-spline expansion QAR(1) model for Melbourne daily temperature is described in Koenker(2000) illustrating this approach.

The statistical properties of nonlinear QAR models and associated estimators are much more complicated than the linear QAR model that we study in the present paper. Despite the possible crossing of quantile curves, we believe that the linear QAR model provides a convenient and useful local approximation to nonlinear QAR models. Such simplied QAR models can still deliver important insight about dynamics, e.g. adjustment asymmetries, in economic time series and thus provides a useful tool in empirical diagnostic time series analysis. 


\section{Inference On The QAR Process}

In this section, we turn our attention to inference in QAR models. Although other inference problems can be analyzed, we consider here the following inference problems that are of paramount interest in many applications. The first hypothesis is the quantile regression analog of the classical representation of linear restrictions on $\theta$ : (1) $H_{01}: R \theta(\tau)=r$, with known $R$ and $r$, where $R$ denotes an $q \times p$-dimensional matrix and $r$ is an $q$-dimensional vector. In addition to the classical inference problem, we are also interested in testing for asymmetric dynamics under the QAR framework. Thus we consider the hypothesis of parameter constancy, which can be formulated in the form of: (2) $H_{02}: R \theta(\tau)=r$, with unknown but estimable $r$. We consider both the cases at specific quantiles $\tau$ (say, median, lower quartile, upper quartile) and the case over a range of quantiles $\tau \in \mathcal{T}$.

5.1. The Regression Wald Process and Related Tests. Under the linear hypothesis $H_{01}: R \theta(\tau)=r$ and the assumptions of Theorem 3, we have

$$
V_{n}(\tau)=\sqrt{n}\left[R \Omega_{1}^{-1} \Omega_{0} \Omega_{1}^{-1} R^{\top}\right]^{-1 / 2}(R \widehat{\theta}(\tau)-r) \Rightarrow B_{q}(\tau),
$$

where $B_{q}(\tau)$ represents a $q$-dimensional standard Brownian Bridge. For any fixed $\tau$, $B_{q}(\tau)$ is $\mathcal{N}\left(0, \tau(1-\tau) I_{q}\right)$. Therefore, the regression Wald process can be constructed as

$$
W_{n}(\tau)=n(R \widehat{\theta}(\tau)-r)^{\top}\left[\tau(1-\tau) R \widehat{\Omega}_{1}^{-1} \widehat{\Omega}_{0} \widehat{\Omega}_{1}^{-1} R^{\top}\right]^{-1}(R \widehat{\theta}(\tau)-r),
$$

where $\widehat{\Omega}_{1}$ and $\widehat{\Omega}_{0}$ are consistent estimators of $\Omega_{1}$ and $\Omega_{0}$. If we are interested in testing $R \theta(\tau)=r$ over $\tau \in \mathcal{T}$, we may consider, say, the following Kolmogorov-Smirnov (KS) type sup-Wald test:

$$
K S W_{n}=\sup _{\tau \in \mathcal{T}} W_{n}(\tau),
$$

If we are interested in testing $R \theta(\tau)=r$ at a particular quantile $\tau=\tau_{0}$, a Chi-square test can be conducted based on the statistic $W_{n}\left(\tau_{0}\right)$. The limiting distributions are summarized in the following Theorem.

Theorem 5.1. Under the assumptions of Theorem 3 and the linear restriction $H_{01}$,

$$
W_{n}\left(\tau_{0}\right) \Rightarrow \chi_{q}^{2}, \text { and } K S W_{n}=\sup _{\tau \in \mathcal{T}} W_{n}(\tau) \Rightarrow \sup _{\tau \in \mathcal{T}} Q_{q}^{2}(\tau),
$$

where $Q_{q}(\tau)=\left\|B_{q}(\tau)\right\| / \sqrt{\tau(1-\tau)}$ is a Bessel process of order $q$, where $\|\cdot\|$ represents the Euclidean norm. For any fixed $\tau, Q_{q}^{2}(\tau) \sim \chi_{q}^{2}$ is a centered Chi-square random variable with q-degrees of freedom. 
5.2. Testing For Asymmetric Dynamics. The hypothesis that $\theta_{j}(\tau), j=1, \cdots$ $\cdot, p$, are constants over $\tau$ (i.e. $\theta_{j}(\tau)=\mu_{j}$ ) can be represented in the form of $H_{02}$ : $R \theta(\tau)=r$ by taking $R=\left[0_{p \times 1} \vdots I_{p}\right]$ and $r=\left[\mu_{1}, \cdots, \mu_{p}\right]^{\prime}$, with unknown parameters $\mu_{1}, \cdots, \mu_{p}$. The Wald process and associated limiting theory provide a natural test for the hypothesis $R \theta(\tau)=r$ when $r$ is known. To test the hypothesis with unknown $r$, appropriate estimator of $r$ is needed. In many econometrics applications, a $\sqrt{n}$ consistent estimator of $r$ is available. If we look at the process

$$
\widehat{V}_{n}(\tau)=\sqrt{n}\left[R \widehat{\Omega}_{1}^{-1} \widehat{\Omega}_{0} \widehat{\Omega}_{1}^{-1} R^{\top}\right]^{-1 / 2}(R \widehat{\theta}(\tau)-\widehat{r})
$$

then under $H_{02}$,

$$
\begin{aligned}
& \widehat{V}_{n}(\tau)=\sqrt{n}\left[R \widehat{\Omega}_{1}^{-1} \widehat{\Omega}_{0} \widehat{\Omega}_{1}^{-1} R^{\top}\right]^{-1 / 2}(R \widehat{\theta}(\tau)-r)-\sqrt{n}\left[R \widehat{\Omega}_{1}^{-1} \widehat{\Omega}_{0} \widehat{\Omega}_{1}^{-1} R^{\top}\right]^{-1 / 2}(\widehat{r}-r) \\
& (12) \quad \Rightarrow B_{q}(\tau)-f\left(F^{-1}(\tau)\right)\left[R \Omega_{0}^{-1} R^{\top}\right]^{-1 / 2} Z
\end{aligned}
$$

where $Z=\lim \sqrt{n}(\widehat{r}-r)$. The necessity of estimating $r$ introduces a drift component $\left(f\left(F^{-1}(\tau)\right)\left[R \Omega_{0}^{-1} R^{\top}\right]^{-1 / 2} Z\right)$ in addition to the simple Brownian bridge process, invalidating the distribution-free character of the original Kolmogorov-Smirnov (KS) test.

To restore the asymptotically distribution free nature of inference, we employ a martingale transformation proposed by Khmaladze (1981) over the process $\widehat{V}_{n}(\tau)$. Denote $d f(x) / d x$ as $\dot{f}$, and define

$$
\dot{g}(r)=\left(1,(\dot{f} / f)\left(F^{-1}(r)\right)\right)^{\top}, \text { and } C(s)=\int_{s}^{1} \dot{g}(r) \dot{g}(r)^{\top} d r,
$$

we construct a martingale transformation $\mathcal{K}$ on $\widehat{V}_{n}(\tau)$ defined as:

$$
\widetilde{V}_{n}(\tau)=\mathcal{K} \widehat{V}_{n}(\tau)=\widehat{V}_{n}(\tau)-\int_{0}^{\tau}\left[\dot{g}_{n}(s)^{\top} C_{n}^{-1}(s) \int_{s}^{1} \dot{g}_{n}(r) d \widehat{V}_{n}(r)\right] d s,
$$

where $\dot{g}_{n}(s)$ and $C_{n}(s)$ are uniformly consistent estimators of $\dot{g}(r)$ and $C(s)$ over $\tau \in \mathcal{T}$, and propose the following Kolmogorov-Smirnov ${ }^{2}$ type test based on the transformed process:

$$
K H_{n}=\sup _{\tau \in \mathcal{T}}\left\|\widetilde{V}_{n}(\tau)\right\|
$$

Under the null hypothesis, the transformed process $\widetilde{V}_{n}(\tau)$ converges to a standard Brownian motion. For more discussions of quantile regression inference based on the martingale transformation approach, see, Koenker and Xiao (2002) and references therein.

\footnotetext{
${ }^{2}$ A Cramer-von-Mises type test based on the transformed process can also be constructed and analysed in a similar way.
} 
We assume the following assumptions on the estimators.

A.4: There exist estimators $\dot{g}_{n}(\tau), \widehat{\Omega}_{0}$ and $\widehat{\Omega}_{1}$ satisfying:

i.: $\sup _{\tau \in}\left|\dot{g}_{n}(\tau)-\dot{g}(\tau)\right|=o_{p}(1)$,

ii.: $\left\|\widehat{\Omega}_{0}-\Omega_{0}\right\|=o_{p}(1),\left\|\widehat{\Omega}_{1}-\Omega_{1}\right\|=o_{p}(1), \sqrt{n}(\widehat{r}-r)=O_{p}(1)$.

Theorem 5.2. Under the assumptions $A .1$ - $A .4$ and the hypothesis $\mathrm{H}_{02}$,

$$
\widetilde{V}_{n}(\tau) \Rightarrow W_{q}(\tau), K H_{n}=\sup _{\tau \in \mathcal{T}}\left\|\widetilde{V}_{n}(\tau)\right\| \Rightarrow \sup _{\tau \in \mathcal{T}}\left\|W_{q}(\tau)\right\|
$$

where $W_{q}(r)$ is a q-dimensional standard Brownian motion.

The martingale transformation is based on function $\dot{g}(s)$ which needs to be estimated. There have been quite a few approaches in estimating the score:

$$
\frac{f^{\prime}}{f}\left(F^{-1}(s)\right) \text {. }
$$

Portnoy and Koenker (1989) studied adaptive estimation and employed kernel-smoothing method in estimating the density and score functions, uniform consistency of the estimators is also discussed. Cox (1985) proposed an elegant smoothing spline approach to the estimation of $f^{\prime} / f$ and $\mathrm{Ng}$ (1995) provided an efficient algorithm for computing this score estimator. Estimation of $\Omega_{0}$ is straightforward: $\widehat{\Omega}_{0}=n^{-1} \sum_{t} x_{t} x_{t}^{\top}$. For the estimation of $\widehat{\Omega}_{1}$, see, inter alia, Koenker and Bassett(1982), Koenker (1994), Powell (1987), and Koenker and Machado (1999) for related discussions.

\section{Monte Carlo}

We conducted a Monte Carlo experiment to examine the effectiveness of inference procedures based on the QAR method. To investigate the finite sample performance of QAR based inference procedures, we examine the empirical size and power of the proposed tests and report the representative results in Tables 1-3. The data in our experiments were generated from model (6), where $u_{t}$ are i.i.d. random variables. We are particularly interested in whether or not the time series $y_{t}$ display asymmetric dynamics. Thus, we consider quantile autoregression (2) with $p=1$ and test the hypothesis that $\alpha_{1}(\tau)=$ constant over $\tau$.

For the tests, we consider the Kolmogorov-Smirnov type test $K H_{n}$ given by (13) for different sample sizes and different innovation distributions. We choose $\mathcal{T}=[0.1,0.9]$. Both the case where $u_{t}$ are standard normal variates and the case that $u_{t}$ are student$t$ distributed variables with 3 degrees of freedom are considered. The number of repetitions is 1000, and two sample sizes are examined: $n=100$, and $n=300$.

When $\alpha_{t}=$ constant, the empirical rejection rates gives the size of test. we report the sizees of this test for three choices of $\alpha_{t}$ : (1) $\alpha_{t}=0.95$; (2) $\alpha_{t}=0.9$; (3) $\alpha_{t}=0.6$. The first two choices of $\alpha_{t}(0.95$ and 0.9$)$ are large and close to unity so that the corresponding time series display cartain degree of (symmetric) persistence. 
Under the alternatives, the processes display asymmetric dynamics. For the choice of alternatives, we considered the following four choices of $\alpha_{t}$,

$$
\begin{aligned}
& \alpha_{t}=\varphi_{1}\left(u_{t}\right)= \begin{cases}1, & u_{t} \geq 0 \\
0.8, & u_{t}<0\end{cases} \\
& \alpha_{t}=\varphi_{2}\left(u_{t}\right)= \begin{cases}0.95, & u_{t} \geq 0 \\
0.8, & u_{t}<0\end{cases} \\
& \alpha_{t}=\varphi_{3}\left(u_{t}\right)=\min \left\{0.5+F_{u}\left(u_{t}\right), 1\right\} \\
& \alpha_{t}=\varphi_{4}\left(u_{t}\right)=\min \left\{0.75+F_{u}\left(u_{t}\right), 1\right\}
\end{aligned}
$$

These alternatives deliver processes with different types of asymmetric (or local) persistency. In particular, when $\alpha_{t}=\varphi_{1}\left(u_{t}\right), \varphi_{3}\left(u_{t}\right), \varphi_{4}\left(u_{t}\right), y_{t}$ display unit root behavior in the presence of positive or large values of innovations, but have a mean reversion tendency with negative shocks. The alternative $\alpha_{t}=\varphi_{2}\left(u_{t}\right)$ has local to (or weak) unit root behavior in the presence of positive of innovations, and behave more stationarily when there are negative shocks.

The construction of tests uses estimators of the density and score. We estimate the density (or sparsity function) using the approach described in the text. For the score function $\dot{g}$, we employ the adaptive kernel estimator of Portnoy and Koenker (1989).

The density estimation exerts important influence on the finite sample performance of our test. Unsuitable bandwidth selection can produce poor estimates. For this reason, we pay particular attention to the bandwidth choice in density estimation. In the experiments, we consider the bandwidth choices suggested by Hall and Sheather (1988) and Bofinger (1975) and rescaled versions of them. A bandwidth rule that Hall and Sheather (1988) suggested based on Edgeworth expansion for studentized quantiles is

$$
h_{H S}=n^{-1 / 3} z_{\alpha}^{2 / 3}\left[1.5 s(t) / s^{\prime \prime}(t)\right]^{1 / 3},
$$

where $z_{\alpha}$ satisfies $\Phi\left(z_{\alpha}\right)=1-\alpha / 2$ for the construction of $1-\alpha$ confidence intervals, and $s(t)=\varphi_{0}(t)^{-1}$. In the absence of other information about the form of $s(\cdot)$, we plug-in the Gaussian model to select bandwidth and obtain

$$
h_{H S}=n^{-1 / 3} z_{\alpha}^{2 / 3}\left[1.5 \phi^{2}\left(\Phi^{-1}(t)\right) /\left(2\left(\Phi^{-1}(t)\right)^{2}+1\right)\right]^{1 / 3} .
$$

Another bandwidth selection has been proposed by Bofinger (1975). The Bofinger bandwidth $h_{B}$ was derived based on minimizing the mean squared error of the density estimator and is of order $n^{-1 / 5}$ :

$$
h_{B}=n^{-1 / 5}\left[4.5 s^{2}(t) /\left(s^{\prime \prime}(t)\right)^{2}\right]^{1 / 5} .
$$

Again, we plug-in the Gaussian density and obtain the following bandwidth that has been widely used in practice

$$
h_{B}=n^{-1 / 5}\left[4.5 \phi^{4}\left(\Phi^{-1}(t)\right) /\left(2\left(\Phi^{-1}(t)\right)^{2}+1\right)^{2}\right]^{1 / 5} .
$$

The Monte Carlo results indicate that the Hall-Sheather bandwidth provides a good lower bound and the Bofinger bandwidth provides a reasonable upper bound 
for bandwidth selection in testing parameter constancy. For this reason, we consider bandwidth values between $h_{H S}$ and $h_{B}$. In particular, we consider rescaled versions of $h_{B}$ and $h_{H S}\left(\theta h_{B}\right.$ and $\delta h_{H S}$ where $0<\theta<1$ and $\delta>1$ are scalars $)$ in our Monte Carlo and representative results are reported. Bandwidth values that are constant over the whole range of quantiles are not recommended. The sampling performance of tests using a constant bandwidth turned out to be poor, and are inferior than bandwidth choices such as the Hall/Sheather or Bofinger bandwidth that varies over the quantiles. For these reason, we focus on bandwidth $h_{B}, h_{H S}, \theta h_{B}$, and $\delta h_{H S}$.

The score function was estimated by the method of Portnoy and Koenker (1989) and we choose the Silverman (1986) bandwidth in our Monte Carlo. Our simulation results show that the test is more affected by the estimation of the density than that of the score. Intuitively, the estimator of the density plays the role of a scalar and thus has the largest influence. The Monte Carlo results also indicates that the method of Portnoy and Koenker (1989) coupled with the Silverman bandwidth has reasonably good performance. Table 1 reports the empirical size and power for the case with Gaussian innovations and sample size $n=100$. Considering the fact that many financial applications have notoriously heavy-tailed behavior we consider processes with heavy-tailed distributions. Table 2 reports results when $u_{t}$ are student- $t$ innovations. The sample size corresponding to Table 2 is still $n=100$. Results in Table 2 confirm that, using the quantile regression based approach, power gain can be obtained in the presence of heavy-tailed disturbances. Experiments based on larger sample sizes are also conductedand Table 3 reports the size and power for the case with Gaussian innovations and sample size $n=300$. Results in Table 3 is qualitatively similar to that of Table 1, but it also shows that, as the sample sizes increase, the tests do have improved size and power properties, corroborating the asymptotic theory. In summary, the Monte Carlo results indicate that, by choosing appropriate bandwidth, the proposed tests have reasonable size and power properties. The test using a rescaled version of Bofinger bandwidth $\left(h=0.6 h_{B}\right)$ yields good performance in all three cases. 


\begin{tabular}{ccccc}
\hline \multicolumn{5}{c}{ Table 1: Testing Constancy of Coefficient $\alpha$} \\
\hline Bandwidth & $h=3 h_{H S}$ & $h=h_{H S}$ & $h=h_{B}$ & $h=0.6 h_{B}$ \\
\hline \multicolumn{5}{c}{ Empirical Size } \\
\hline$\alpha_{t}=0.95$ & 0.073 & 0.287 & 0.018 & 0.056 \\
\hline$\alpha_{t}=0.9$ & 0.073 & 0.275 & 0.01 & 0.046 \\
\hline$\alpha_{t}=0.6$ & 0.07 & 0.287 & 0.012 & 0.052 \\
\hline \multicolumn{5}{c}{ Empirical Power } \\
\hline$\alpha_{t}=\varphi_{1}\left(u_{t}\right)$ & 0.474 & 0.795 & 0.271 & 0.391 \\
\hline$\alpha_{t}=\varphi_{2}\left(u_{t}\right)$ & 0.262 & 0.620 & 0.121 & 0.234 \\
\hline$\alpha_{t}=\varphi_{3}\left(u_{t}\right)$ & 0.652 & 0.939 & 0.322 & 0.533 \\
\hline$\alpha_{t}=\varphi_{4}\left(u_{t}\right)$ & 0.159 & 0.548 & 0.046 & 0.114 \\
\hline \multicolumn{5}{c}{} \\
\hline$u_{t}=\mathrm{N}(0,1), n=100$, Number of replication $=1000$ \\
\hline
\end{tabular}

\begin{tabular}{|c|c|c|c|c|}
\hline \multicolumn{5}{|c|}{ Table 2: Testing Constancy of Coefficient $\alpha$} \\
\hline & $h=3 h_{H S}$ & $h=h_{H S}$ & $h=h_{B}$ & $h=0.6 h_{B}$ \\
\hline \multicolumn{5}{|c|}{ Empirical Size } \\
\hline$\alpha_{t}=0.95$ & 0.086 & 0.339 & 0.011 & 0.059 \\
\hline$\alpha_{t}=0.9$ & 0.072 & 0.301 & 0.015 & 0.043 \\
\hline$\alpha_{t}=0.6$ & 0.072 & 0.305 & 0.013 & 0.038 \\
\hline \multicolumn{5}{|c|}{ Empirical Power } \\
\hline$\alpha_{t}=\varphi_{1}\left(u_{t}\right)$ & 0.556 & 0.819 & 0.319 & 0.444 \\
\hline$\alpha_{t}=\varphi_{2}\left(u_{t}\right)$ & 0.348 & 0.671 & 0.174 & 0.279 \\
\hline$\alpha_{t}=\varphi_{3}\left(u_{t}\right)$ & 0.713 & 0.933 & 0.346 & 0.55 \\
\hline$\alpha_{t}=\varphi_{4}\left(u_{t}\right)$ & 0.284 & 0.685 & 0.061 & 0.162 \\
\hline
\end{tabular}




\begin{tabular}{|c|c|c|c|c|}
\hline \multicolumn{5}{|c|}{ Table 3: Testing Constancy of Coefficient $\alpha$} \\
\hline & $h=3 h_{H}$ & $h=h_{H}$ & $h=h_{B}$ & $=0.6 h_{B}$ \\
\hline \multicolumn{5}{|c|}{ Empirical Size } \\
\hline$\alpha_{t}=0.95$ & 0.081 & 0.191 & 0.028 & 0.049 \\
\hline$\alpha_{t}=0.9$ & 0.098 & 0.189 & 0.03 & 0.056 \\
\hline$\alpha_{t}=0.6$ & 0.097 & 0.16 & 0.02 & 0.045 \\
\hline \multicolumn{5}{|c|}{ Empirical Power } \\
\hline$\alpha_{t}=\varphi_{1}\left(u_{t}\right)$ & 0.974 & 0.992 & 0.921 & 0.937 \\
\hline$\alpha_{t}=\varphi_{2}\left(u_{t}\right)$ & 0.831 & 0.923 & 0.685 & 0.763 \\
\hline$\alpha_{t}=\varphi_{3}\left(u_{t}\right)$ & 0.998 & 1 & 0.971 & 0.989 \\
\hline$\alpha_{t}=\varphi_{4}\left(u_{t}\right)$ & 0.557 & 0.897 & 0.235 & 0.392 \\
\hline
\end{tabular}

\section{Empirical Applications}

There have been many claims and observations that some economic time series are asymmetric. For example, it has been observed that increases in the unemployment rate are sharper than declines; firms are more apt to increase than to decrease in prices. It has also been argued that positive shocks to the economy may be more persistent than negative shocks. For this reason, studies have been conducted on the existence of asymmetric behavior in these series. If an economic time series displays asymmetric dynamics systematically, then appropriate models are needed to incorporate such behavior. In this section, we apply the QAR model to two economic time series: unemployment rates and retail gasoline prices in the US. Our empirical analysis indicate that both series display asymmetric dynamics.

7.1. Unemployment Rate. Many studies on unemployment suggest that the response of unemployment to expansionary or contractionary shocks may be asymmetric. An asymmetric response to different types of shocks has important implications in policy. In this section, we examine unemployment dynamics using the proposed procedures.

The data that we consider are quarterly and annual rates of unemployment in the US. In particular, we looked at (seasonally adjusted) quarterly rates, starting from the first quarter of 1948 and ending at the last quarter of 2003, with 224 observations. and the annual rates are from 1890 to 1996. Many empirical studies in the unit root literature have investigated unemployment rate data. Nelson and Plosser (1982) studied the unit root property of annual US unemployment rates in their seminal work on fourteen macroeconomic time series. Evidence based on the unit root tests suggests that the series is stationary. This series and other type unemployment rates have been often re-examined in later analysis. 
We first apply regression (10) on the unemployment rates. We use the BIC criterion of Schwarz (1978) and Rissanen (1978) in selecting the appropriate lag length of the autoregressions. The selected lag length is $p=3$ for the annual data and $p=2$ for the quarterly data. The OLS estimation of the largest autoregressive root is 0.718 for the annual series and 0.941 for the quarterly rates. Quantile autoregression was also performed for each deciles. The estimates of the largest autoregressive root at each quantile is reported in Table 4 . These estimated values are different over different quantiles, displaying asymmetric dynamics over the business cycle. In particular, we find that in the presence of negative shocks, the estimated autoregressive root is generally larger.

\begin{tabular}{|c|c|c|c|c|c|c|c|c|c|}
\hline$\tau$ & 0.1 & 0.2 & 0.3 & 0.4 & 0.5 & 0.6 & 0.7 & 0.8 & 0.9 \\
\hline & \multicolumn{9}{|c|}{ Annual Data } \\
\hline \multirow[t]{2}{*}{$\delta_{0}(\tau)$} & 0.7406 & 0.7765 & 0.9293 & 0.8710 & 0.8588 & 0.7933 & 0.7270 & 0.6808 & 0.5991 \\
\hline & \multicolumn{9}{|c|}{ Quarterly Data } \\
\hline$\overline{\left.\delta_{0} \tau\right)}$ & 0.912 & 0.908 & 0.931 & 0.919 & 0.951 & 0.959 & 0.967 & 0.962 & 0.953 \\
\hline
\end{tabular}

We then test asymmetric dynamics using the martingale transformation based Kolmogorov-Smirnov procedure (13) based on quantile autoregression (8). According to the suggestion from the Monte Carlo results, we choose the rescaled Hall and Sheather (1988) bandwidth $3 h_{H S}$ and the rescaled Bofinger (1975) bandwidth $0.6 h_{B}$ in estimating the density function. The tests were constructed over $\tau \in \mathcal{T}=[0.05,0.95]$ and results are reported in Table 5. The empirical results indicate that asymmetric behavior exist in these series.

\begin{tabular}{cccc||c}
\hline \multicolumn{2}{c|}{ Table 5: The Kolmogrov Test $\sup _{\tau \in \mathcal{T}}$} & $\widetilde{V}_{n}(\tau)$ & \\
\hline Bandwidth & $0.6 h_{B}$ & $3 h_{H S}$ & $5 \%$ Critical Values \\
\hline Annual Rate & 4.8962 & 5.1172 & 4.523 \\
\hline Quarterly Rate & 4.4599 & 5.3637 & 3.393 \\
\hline
\end{tabular}

7.2. Retail Gasoline Price Dynamics. Our second application investigates the asymptotic price dynamics in the retail gasoline market. It has been documented that many markets exist asymmetric price dynamics. In this section, we apply the QAR madel to the US retail gasoline prices and investigate the existence of asymmetric price adjustment. We consider weekly data of US regular gasoline retail price from August 20, 1990 to Februry 16, 2004. The sample size is 699.

Table 7 report the OLS based augmented Dickey-Fuller regression estimation results and the ADF tests for the null hypothesis of a unit root (again we use the BIC criterion to select the lag length $(p=4)$ of the autoregressions.) The evidence we obtain is marginal; the unit root null is rejected by the coefficient based test $A D F_{\alpha}$, but can not be rejected by the $t$ ratio based test $A D F_{t}$. 
We next consider quantile regression based on the ADF model (9) to examine the persistency behavior of the gasoline price series at various quantiles. In particular, Table 6 reports the estimates of the largest autoregressive roots $\widehat{\delta}_{0}(\tau)$ at each decile. The evidence based on these point estimates of the largest autoregressive root at each quantile suggests that the gasoline price series has asymmetric dynamics. From the table we can see that there exists asymmetry in persistency. The largest autoregressive coefficient estimate $\widehat{\delta}_{0}(\tau)$ has different values over different quantiles, displaying asymmetric dynamics over the business cycle. In particular, $\widehat{\delta}_{0}(\tau)$ monotonically increases when we move from lower quantiles to higher quantiles. The autoregressive coefficient values at the lower quantiles are relatively small, indicating that the local behavior of the gasoline price would be stationary. However, at higher quantiles, the largest autoregressive root is close to or even slightly above unity, consequently the time series display unit root or locally explosive behavior at upper quantiles.

\begin{tabular}{|c|c|c|c|c|c|c|c|c|}
\hline Table & 6: The Estim & ated Lc & gest $A$ & R Roo & at $\mathrm{Ea}$ & h Dec & le of $\mathrm{Ga}$ & ine \\
\hline$\tau$ & $\begin{array}{ll}0.1 & 0.2\end{array}$ & 0.3 & 0.4 & 0.5 & 0.6 & 0.7 & 0.8 & 0 . \\
\hline$\delta_{0}(\tau)$ & 0.958 & 0.971 & 0.980 & 0.996 & 1.005 & 1.016 & 1.024 & 1.0 \\
\hline & & & ble 7: & Unit R & ot Tes & & & \\
\hline & & & & est Sta & istic & $5 \%($ & ritical V & les \\
\hline & OLS Based $A$ & $\overline{D F_{\alpha} \text { tt }}$ & & -17.1 & & & -14.1 & \\
\hline & OLS Based $A$ & $\overline{D F_{t}}$ te & & -2.67 & & & -2.86 & \\
\hline & $\sup _{\tau \in \mathcal{T}} \| V$ & $n(\tau)$ & & 8.347 & & & 5.560 & \\
\hline & $Q K S$ & & & 35.79 & & & 13.2181 & \\
\hline & $\overline{Q C M}$ & & & $\overline{320.4}$ & & & 19.7209 & \\
\hline
\end{tabular}

We next perform formal tests for the null hypothesis that the gasoline price series has constant autoregressive coefficents. We apply the martingale transformation based Kolmogorov-Smirnov procedure (13) based on quantile autoregression (2), constancy of coefficients is rejected. The calculated Kolmogorov-Smirnov statistic (using the rescaled Bofinger (1975) bandwidth $0.6 h_{B}$ is 8.347735 (lag length $p=4$ ), which is larger than the $5 \%$ level critical value (5.56). However, taking into account of the unit root behavior under the null, we consider the following (coefficient-based) empirical quantile process

$$
U_{n}(\tau)=n\left(\widehat{\delta}_{0}(\tau)-1\right)
$$

and the Kolmogorov-Smirnov (KS) or Cramer-von-Mises (CvM) type tests:

$$
Q K S_{\alpha}=\sup _{\tau \in \mathcal{T}}\left|U_{n}(\tau)\right|, Q C M_{\alpha}=\int_{\tau \in \mathcal{T}} U_{n}(\tau)^{2} d \tau
$$


Using the results of unit root quantile regression asymptotics provided by Koenker and Xiao (2003), we have, under the unit root hypothesis,

$$
U_{n}(\tau) \Rightarrow U(\tau)=\frac{1}{f\left(F^{-1}(\tau)\right)}\left[\int_{0}^{1} \underline{B}_{y}^{2}\right]^{-1} \int_{0}^{1} \underline{B}_{y} d B_{\psi}^{\tau} .
$$

where $\underline{B}_{w}(r)$ and $B_{\psi}^{\tau}(r)$ are limiting processes of $n^{-1 / 2} \sum_{t=1}^{[n r]} \Delta y_{t}$ and $\left.n^{-1 / 2} \sum_{t=1}^{[n r]} \psi_{\tau}\left(u_{t \tau}\right)\right)$. We adopt the approach of Koenker and Xiao (2003) and approximate the distributions of the limiting variates by resampling method and construct bootstrap tests for the unit root hypothesis based on (14).

Table 7 reports the $Q K S_{\alpha}$ and $Q C M_{\alpha}$ tests for the null hypothesis of a constant unit root. The $5 \%$ level critical values calculated based on the resampling procedure are also reported in the table. The constant unit root hypothesis is rejected at $5 \%$ level by both tests. These results, together with the point estimates reported in Table 6 , indicate that the gasoline price series has asymmetric adjustment dynamics and thus is not well characterized as a constant coefficient unit root process. 


\section{Appendix: Proofs}

8.1. Proof of Theorem 1. Giving a $p$-th order autoregression process (5), we denote $E\left(\alpha_{j, t}\right)=\mu_{j}$, and assume that $1-\sum \mu_{j} \neq 0$. Let $\mu=\mu_{0} /\left(1-\sum_{j=1}^{p} \mu_{j}\right)$, and denote

$$
\underline{y}_{t}=y_{t}-\mu
$$

we have

$$
\underline{y}_{t}=\alpha_{1, t} \underline{y}_{t-1}+\cdots+\alpha_{p, t} \underline{y}_{t-p}+v_{t}
$$

where

$$
v_{t}=u_{t}+\mu \sum_{l=1}^{p}\left(\alpha_{l, t}-\mu_{l}\right) .
$$

It's easy to see that $E v_{t}=0$ and $E v_{t} v_{s}=0$ for any $t \neq s$ since $E \alpha_{l, t}=\mu_{l}$ and $u_{t}$ are independent. In order to derive stationarity conditions for the process $\underline{y}_{t}$, we first find an $\mathcal{F}_{t}$-measurable solution for (16). We define the $p \times 1$ random vectors

$$
\underline{Y}_{t}=\left[\underline{y}_{t}, \cdots \cdot \underline{y}_{t-p+1}\right]^{\prime}, V_{t}=\left[v_{t}, 0, \cdots, 0\right]^{\prime}
$$

and the $p \times p$ random matrix

$$
A_{t}=\left[\begin{array}{cc}
A_{p-1, t} & \alpha_{p, t} \\
I_{p-1} & 0_{p-1}
\end{array}\right]
$$

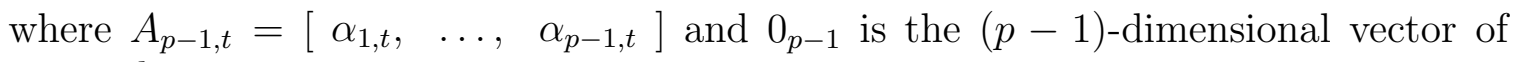
zeros, then

$$
E\left(V_{t} V_{t}^{\prime}\right)=\left[\begin{array}{cc}
\sigma_{v}^{2} & 0_{1 \times(p-1)} \\
0_{(p-1) \times 1} & 0_{(p-1) \times(p-1)}
\end{array}\right]=\Sigma
$$

and the original process can be written as

$$
\underline{Y}_{t}=A_{t} \underline{Y}_{t-1}+V_{t}
$$

By substitution, we have

$$
\begin{aligned}
\underline{Y}_{t} & =V_{t}+A_{t} V_{t-1}+A_{t} A_{t-1} V_{t-2}+\left[A_{t} \cdots A_{t-m+1}\right] V_{t-m}+\left[A_{t} \cdots A_{t-m}\right] \underline{Y}_{t-m-1} \\
& =\underline{Y}_{t, m}+R_{t, m}
\end{aligned}
$$

where

$$
\underline{Y}_{t, m}=\sum_{j=0}^{m} B_{j} V_{t-j}, R_{t, m}=B_{m+1} \underline{Y}_{t-m-1}, \text { and } B_{j}=\left\{\begin{array}{l}
\prod_{l=0}^{j-1} A_{t-l}, j \geq 1 . \\
I, j=0 .
\end{array} .\right.
$$

The stationarity of an $\mathcal{F}_{t}$-measurable solution for $y_{t}$ involves the convergence of $\left\{\sum_{j=0}^{m} B_{j} V_{t-j}\right\}$ and $\left\{R_{t, m}\right\}$ as $m$ increases, for fixed $t$. Following a similar analysis as Nicholls and Quinn (1982, Chapter 2), We need to verify that vecE $\left[\underline{Y}_{t, m} \underline{Y}_{t, m}^{\prime}\right]$ converges as $m \rightarrow \infty$. Notice that $B_{j}$ is independent with $V_{t-j}$ and $\left\{u_{t}, t=0, \pm 1, \pm 2, \cdots\right\}$ 
are independent random variables, thus, $\left\{B_{j} V_{t-j}\right\}_{j=0}^{\infty}$ is an orthogonal sequence in the sense that $E\left[B_{j} V_{t-j} B_{k} V_{t-k}\right]=0$ for any $j \neq k$. Thus

$$
\operatorname{vec} E\left[\underline{Y}_{t, m} \underline{Y}_{t, m}^{\prime}\right]=\operatorname{vec} E\left[\left(\sum_{j=0}^{m} B_{j} V_{t-j}\right)\left(\sum_{j=0}^{m} B_{j} V_{t-j}\right)^{\prime}\right]=\operatorname{vec} E\left[\sum_{j=0}^{m} B_{j} V_{t-j} V_{t-j}^{\prime} B_{j}^{\prime}\right]
$$

Notice that $\operatorname{vec}(A B C)=\left(C^{\prime} \otimes A\right) \operatorname{vec}(B)$, and $\left(\prod_{l=0}^{j} A_{l}\right) \otimes\left(\prod_{k=0}^{j} B_{k}\right)=\prod_{k=0}^{j}\left(A_{k} \otimes\right.$ $B_{k}$ ), we have

$$
\begin{aligned}
\operatorname{vec} E\left[\sum_{j=0}^{m} B_{j} V_{t-j} V_{t-j}^{\prime} B_{j}^{\prime}\right] & =E\left[\sum_{j=0}^{m}\left(B_{j} \otimes B_{j}\right) \operatorname{vec}\left(V_{t-j} V_{t-j}^{\prime}\right)\right] \\
& =E\left[\sum_{j=0}^{m}\left(\prod_{l=0}^{j-1} A_{t-l}\right) \otimes\left(\prod_{l=0}^{j-1} A_{t-l}\right) \operatorname{vec}\left(V_{t-j} V_{t-j}^{\prime}\right)\right] \\
& =\sum_{j=0}^{m} \prod_{l=0}^{j-1} E\left(A_{t-l} \otimes A_{t-l}\right) \operatorname{vec} E\left(V_{t-j} V_{t-j}^{\prime}\right)
\end{aligned}
$$

If we denote

$$
A=E\left[A_{t}\right]=\left[\begin{array}{cc}
\bar{\mu}_{p-1} & \alpha_{p} \\
I_{p-1} & 0_{p-1}
\end{array}\right]
$$

where $\bar{\mu}_{p-1}=\left[\begin{array}{lll}\alpha_{1}, & \ldots, \alpha_{p-1}\end{array}\right]$, then $A_{t}=A+\Xi_{t}$, where $E\left(\Xi_{t}\right)=0$, and

$$
E\left(A_{t-l} \otimes A_{t-l}\right)=E\left[\left(A+\Xi_{t}\right) \otimes\left(A+\Xi_{t}\right)\right]=A \otimes A+\mathrm{E}\left(\Xi_{t} \otimes \Xi_{t}\right)=\Omega_{A}
$$

then

$$
\operatorname{vec} E\left[\left(\sum_{j=0}^{m} B_{j} V_{t-j}\right)\left(\sum_{j=0}^{m} B_{j} V_{t-j}\right)^{\prime}\right]=\sum_{j=0}^{m} \Omega_{A}^{j} \operatorname{vec}(\Sigma) .
$$

The critical condition for the stationarity of the process $\underline{y}_{t}$ is that $\sum_{j=0}^{m} \Omega_{A}^{j}$ converges as $m \rightarrow \infty$.

The matrix $\Omega_{A}$ may be represented in Jordan canonical form as $\Omega_{A}=P \Lambda P^{-1}$, where $\Lambda$ has the eigenvalues of $\Omega_{A}$ along its main diagonal. If the eigenvalues of $\Omega_{A}$ have moduli less than unity, $\Lambda^{j}$ converges to zero at a geometric rate. Notice that $\Omega_{A}^{j}=P \Lambda^{j} P^{-1}$, following a similar analysis as Nicholls and Quinn (1982, Chapter 2), $\underline{Y}_{t}$ (and thus $\left.y_{t}\right)$ is stationary and can be represented as

$$
\underline{Y}_{t}=\sum_{j=0}^{\infty} B_{j} V_{t-j}
$$

The central limit theorem then follows from Billingsley (1961) (also see Nicholls and Quinn (1982, Theorem A.1.4)). 
8.2. Proof of Theorem 3. If we denote $\widehat{v}=\sqrt{n}(\widehat{\theta}(\tau)-\theta(\tau))$, then $\rho_{\tau}\left(y_{t}-\widehat{\theta}(\tau)^{\top} x_{t}\right)=$ $\rho_{\tau}\left(u_{t \tau}-\left(n^{-1 / 2} \widehat{v}\right)^{\top} x_{t}\right)$, where $u_{t \tau}=y_{t}-x_{t}^{\top} \theta(\tau)$. Minimization of (8) is equivalent to minimizing:

$$
Z_{n}(v)=\sum_{t=1}^{n}\left[\rho_{\tau}\left(u_{t \tau}-\left(n^{-1 / 2} v\right)^{\top} x_{t}\right)-\rho_{\tau}\left(u_{t \tau}\right)\right]
$$

If $\widehat{v}$ is a minimizer of $Z_{n}(v)$, we have $\widehat{v}=\sqrt{n}(\widehat{\theta}(\tau)-\theta(\tau))$. The objective function $Z_{n}(v)$ is a convex random function. Knight (1989) (also see Pollard (1991) and Knight (1998)) shows that if the finite-dimensional distributions of $Z_{n}(\cdot)$ converge weakly to those of $Z(\cdot)$ and $Z(\cdot)$ has a unique minimum, the convexity of $Z_{n}(\cdot)$ implies that $\widehat{v}$ converges in distribution to the minimizer of $Z(\cdot)$.

We use the following identity: if we denote $\psi_{\tau}(u)=\tau-I(u<0)$, for $u \neq 0$,

$$
\begin{aligned}
\rho_{\tau}(u-v)-\rho_{\tau}(u) & =-v \psi_{\tau}(u)+(u-v)\{I(0>u>v)-I(0<u<v)\} \\
& =-v \psi_{\tau}(u)+\int_{0}^{v}\{I(u \leq s)-I(u<0)\} d s .
\end{aligned}
$$

Thus the objective function of minimization problem can be written as

$$
\begin{aligned}
& \sum_{t=1}^{n}\left[\rho_{\tau}\left(u_{t \tau}-\left(n^{-1 / 2} v\right)^{\prime} x_{t}\right)-\rho_{\tau}\left(u_{t \tau}\right)\right] \\
= & -\sum_{t=1}^{n}\left(n^{-1 / 2} v\right)^{\prime} x_{t} \psi_{\tau}\left(u_{t \tau}\right)+\sum_{t=1}^{n} \int_{0}^{\left(n^{-1 / 2} v\right)^{\prime} x_{t}}\left\{I\left(u_{t \tau} \leq s\right)-I\left(u_{t \tau}<0\right)\right\} d s
\end{aligned}
$$

We first consider the limiting behavior of

$$
W_{n}(v)=\sum_{t=1}^{n} \int_{0}^{\left(n^{-1 / 2} v\right)^{\prime} x_{t}}\left\{I\left(u_{t \tau} \leq s\right)-I\left(u_{t \tau}<0\right)\right\} d s .
$$

For convenience of asymptotic analysis, we denote

$$
W_{n}(v)=\sum_{t=1}^{n} \xi_{t}(v), \xi_{t}(v)=\int_{0}^{\left(n^{-1 / 2} v\right)^{\prime} x_{t}}\left\{I\left(u_{t \tau} \leq s\right)-I\left(u_{t \tau}<0\right)\right\} d s .
$$

We further define $\bar{\xi}_{t}(v)=E\left\{\xi_{t}(v) \mid \mathcal{F}_{t-1}\right\}$, and $\bar{W}_{n}(v)=\sum_{t=1}^{n} \bar{\xi}_{t}(v)$, then $\left\{\xi_{t}(v)-\right.$ $\left.\bar{\xi}_{t}(v)\right\}$ is a martingale difference sequence.

Notice that

$$
u_{\tau t}=y_{t}-x_{t}^{\prime} \alpha(\tau)=y_{t}-F_{t-1}^{-1}(\tau)
$$




$$
\begin{aligned}
\bar{W}_{n}(v) & =\sum_{t=1}^{n} E\left\{\int_{0}^{\left(n^{-1 / 2} v\right)^{\prime} x_{t}}\left[I\left(u_{t \tau} \leq s\right)-I\left(u_{t \tau}<0\right)\right] \mid \mathcal{F}_{t-1}\right\} \\
& =\sum_{t=1}^{n} \int_{0}^{\left(n^{-1 / 2} v\right)^{\prime} x_{t}}\left[\int_{F_{t-1}^{-1}(\tau)}^{s+F_{t-1}^{-1}(\tau)} f_{t-1}(r) d r\right] d s \\
& =\sum_{t=1}^{n} \int_{0}^{\left(n^{-1 / 2} v\right)^{\prime} x_{t}}\left[\frac{F_{t-1}\left(s+F_{t-1}^{-1}(\tau)\right)-F_{t-1}\left(F_{t-1}^{-1}(\tau)\right)}{s}\right] s d s
\end{aligned}
$$

Under assumption A.3,

$$
\begin{aligned}
\bar{W}_{n}(v) & =\sum_{t=1}^{n} \int_{0}^{n^{-1 / 2} v^{\prime} x_{t}} f_{t-1}\left(F_{t-1}^{-1}(\tau)\right) s d s+o_{p}(1) \\
& =\frac{1}{2 n} \sum_{t=1}^{n} f_{t-1}\left(F_{t-1}^{-1}(\tau)\right) v^{\prime} x_{t} x_{t}^{\prime} v+o_{p}(1)
\end{aligned}
$$

By our assumptions and stationarity of $y_{t}$, we have

$$
\bar{W}_{n}(v) \Rightarrow \frac{1}{2} v^{\prime} \Omega_{1} v
$$

Using the same argument as Herce(1996), the limiting distribution of $\sum_{t} \xi_{t}(v)$ is the same as that of $\sum_{t} \bar{\xi}_{t}(v)$.

For the behavior of the first term, $n^{-1 / 2} \sum_{t=1}^{n} x_{t} \psi_{\tau}\left(u_{t \tau}\right)$, in the objective function, notice that $x_{t} \in \mathcal{F}_{t-1}$ and $E\left[\psi_{\tau}\left(u_{t \tau}\right) \mid \mathcal{F}_{t-1}\right]=0, x_{t} \psi_{\tau}\left(u_{t \tau}\right)$ is a martingale difference sequence and thus $n^{-1 / 2} \sum_{t=1}^{n} x_{t} \psi_{\tau}\left(u_{t \tau}\right)$ satisfies a central limit theorem. Following the arguments of Portnoy (1984) and Gutenbrunner and Jurevckova (1992), the autoregression quantile process is tight and thus the limiting variate viewed as a random function of $\tau$, is a Brownian bridge over $\tau \in \mathcal{T}$,

$$
n^{-1 / 2} \sum_{t=1}^{n} x_{t} \psi_{\tau}\left(u_{t \tau}\right) \Rightarrow \Omega_{0}^{1 / 2} B_{k}(\tau) .
$$

For each fixed $\tau, n^{-1 / 2} \sum_{t=1}^{n} x_{t} \psi_{\tau}\left(u_{t \tau}\right)$ converges to a $q$-dimensional vector normal variate with covariance matrix $\tau(1-\tau) \Omega_{0}$. Thus,

$$
\begin{aligned}
& Z_{n}(v) \\
= & \sum_{t=1}^{n}\left[\rho_{\tau}\left(u_{t \tau}-\left(n^{-1 / 2} v\right)^{\prime} x_{t}\right)-\rho_{\tau}\left(u_{t \tau}\right)\right] \\
= & -\sum_{t=1}^{n}\left(n^{-1 / 2} v\right)^{\prime} x_{t} \psi_{\tau}\left(u_{t \tau}\right)+\sum_{t=1}^{n} \int_{0}^{\left(n^{-1 / 2} v\right)^{\prime} x_{t}}\left\{I\left(u_{t \tau} \leq s\right)-I\left(u_{t \tau}<0\right)\right\} d s . \\
\Rightarrow & -v^{\prime} \Omega_{0}^{1 / 2} B_{k}(\tau)+\frac{1}{2} v^{\prime} \Omega_{1} v=Z(v)
\end{aligned}
$$


By the convexity Lemma of Pollard (1991) and arguments of Knight (1989), notice that $Z_{n}(v)$ and $Z(v)$ are minimized at $\widehat{v}=\sqrt{n}(\widehat{\alpha}(\tau)-\alpha(\tau))$ and $\Sigma^{1 / 2} B_{k}(\tau)$ respectively, by Lemma A of Knight (1989) we have,

$$
\Sigma^{-1 / 2} \sqrt{n}(\widehat{\alpha}(\tau)-\alpha(\tau)) \Rightarrow B_{k}(\tau)
$$

\section{REFERENCES}

[1] Balke, N. and T. Fomby, 1997, Threshold Cointegration, International Economic Review, 38, 627-645.

[2] Bassett, G., and R. Koenker, 1982, An Empirical Quantile Function for linear models with iid errors, Journal of the American Statistical Association, Vol. 77, 407-415.

[3] Beaudry, P. and G. Koop, 1993, Do recessions permanently change output?, Journal of Monetary Economics, 31, 149-163.

[4] Billingsley, P, 1961, The Lindeberg-Levy Theorem for Martingales, Proc. Amer. Math. Soc., 12, 788-792.

[5] Bradley, M.D. and D.W. Jansen, 1997, Nonlinear business cycle dynamics: Cross-country evidence on the persistence of aggregate shocks, Economic Inquiry, 35, 495-509.

[6] Brandt, A., 1986, The stochastic equation $Y_{n+1}=A_{n} Y_{n}+B_{n}$ with stationary coefficients, Adv. Applied Probability, 18, 211-220.

[7] Bofinger, E., 1975, Estimation of a density function using order statistics," Australian Journal of Statistics, 17, 1-7.

[8] Caner, M. and B. Hansen, Threshold Autoregression with a unit root, 2001, Econometrica.

[9] Cox, D., 1985, A Penalty Method for Nonparametric Estimation of the Logarithmic Derivative of a Density Function," Annals of Institute of Mathematical Statistics, 37, 271-288.

[10] Delong, J.B., and Summers, L.H., 1986, Are business cycle symmetrical?, in Gordon, R.J. (ed.), American Business Cycle, Chicago University Press, Chicago.

[11] Denneberg, D. 1994, Non-additive measure and integral, Kluwer Academic Publishers.

[12] Enders, W. and C. Granger, Unit Root tests and asymetric adjustment with an example sing the term structure of interest rates, Journal of Business and Economic Statistics, V. 16, No. 3, 304-311.

[13] Evans, M. and P. Wachtel, 1993, Inflation regioms and the sources of inflation uncertainty, Journal of Money, Credit, and Banking, 25, 475-511.

[14] Gonzalez, M. and J. Gonzalo, Threshold unit root models, Working paper, U. Carlos III de Madrid.

[15] Gutenbrunner, C., and Jureckova, J. (1992), "Regression Rank Scores and Regression Quantiles," Annals of Statistics, 20, 305-330.

[16] Hall, P., and S. Sheather, 1988, On the distribution of a studentized quantile," JRSS(B), 50, 381-391.

[17] Hamilton, J., 1989, A new approach to the economic analysis of nonstationary time series and the business cycle, Econometrica, 57, 357-384.

[18] Hansen, B., 2000, Sample splitting and Threshold estimation, Econometrica, 68, 575-603.

[19] Hess, G.D. and Iwata, 1997, Asymmetric persistence in GDP? A deeper look at depth, Journal of Monetary Economics, 40, 535-554.

[20] Hasan, M.N. and R. Koenker, 1997, Robust rank tests of the unit root hypothesis, Econometrica 65, No.1, 133-161.

[21] Herce, M., 1996, Asymptotic Theory od LAD estimation in a unit root process with finite variance errors, Econometric Theory, 12, 129-153. 
[22] Jureckova and Hallin, 1999, Optimal Tests for Autoregressive Models Based on Autoregression Rank Scores, The Annals of Statistics, 27, 1385-1414,

[23] Karlsen, H.A., 1990, Existence of Moments in a stationary stochastic difference equation, Adv. Applied Probability, 22, 129-146.

[24] Khmaladze, E., 1981, Martingale Approach to the goodness of fit tests, Theory Probab. Appl., 26, 246-265.

[25] Knight, K., 1989, Limit Theory for Autoregressive-parameter estimates in an infinite -variance random walk, The Canadian Journal of Statistics, V. 17, 261-278.

[26] Knight, K., 1998, Asymptotics for L1 regression estimates under general conditions, Annals of Statistics.

[27] Koenker, R., 1994, Confidence Intervals for regression quantiles," in Proc. of the 5th Pradue Symp. on Asym. Stat., pp349-359, Springer-Verlag.

[28] Koenker, R. and G. Bassett, 1978, Regression Quantiles, Econometrica, V46, 33-49.

[29] Koenker, R. and J. Machado, 1999, Goodness of fit and relatedinference processes for quantile regression, Journal of the American Statistical Association, Vol. 81, 1296-1310.

[30] Koenker, R. 2000, Galton, Edgeworth, Frisch and prospects for quantile regression in econometrics, J. of Econometrics, 95, 347-374.

[31] Koenker, R. and Z. Xiao, 2002, Inference on the Quantile Regression Processes, Econometrica, 70, 1583-1612.

[32] Koenker, R. and Z. Xiao, 2003, Unit Root Quantile Regression Inference, Journal of the American Statistical Association, forthcoming

[33] Koul, H., and A. K. Saleh, 1995, Autoregression quantiles and related rank-scores processes, The Annals of Statistics, V. 23, No. 2, 670-689.

[34] Kuan, C.M. and Y.L. Huang, 2001, The semi-nonstationary process: Model and empirical evidence, preprint.

[35] Linton, O. and Y.-J. Whang, 2004, A Quantilogram Approach to Evaluating Directional Predictability, preprint.

[36] Neftci, S., 1984, Are economic time series asymmetric over the business cycle?, Journal of Political Economy, V.92, 307-328.

[37] Nelson, C.R. and C.I. Plosser, 1982, Trends and random walks in macroeconomic time series: some evidence and implications, Journal of Monetary Economics, 10, 139-162.

[38] Ng, P., 1994, Smoothing Spline Score Estimation," SIAM Journal of Scientific and Statistical Computing, 15, 1003-1025.

[39] Nicholls, D.F., and B.G. Quinn, 1982, Random Coefficient Autoregressive Models: An Introduction, Springer-Verlag.

[40] Pollard, D., 1991, Asymptotics for Least Absolute Deviation Regression Estimators, Econometric Theory, 7, 186-199.

[41] Portnoy, S, (1984), "Tightness of the Sequence of Empiric cdf Processes Defined from Regression Fractiles," in Robust and Nonlinear Time Series Analysis, eds. J. Franke, W. Hardle, and D. Martin, Springer-Verlag: New York.

[42] Portnoy, S., and R. Koenker, 1989, Adaptive L-estimation of linear models," Annals of Statistics, $17,362-381$.

[43] Pourahmadi, M., 1986, On stationarity of the solution of a doubly stochastic model, J. of Time Series Analysis, 7, 123-131.

[44] Powell, J., 1989, Estimation of monotonic regression models under quantile restrictions," in Nonparametric and semiparametric methods in econometrics, J. Powell, G. Tauchen, (ed.), Cambridge University Press.

[45] Rissanen, J., 1978, Modelling by shortest data description, Automatica, 14, 465-471. 
[46] Schmeidler, D., 1996, Integral representation without additivity, Proceedings of Amer. Math. Society, 97, 255-261.

[47] Schwarz, G., 1978, Estimating the dimension of a model, The Annals of Statistics, 6, 461-464.

[48] Silverman, B., 1986, Density Estimation for statistics and data analysis, Chapman and Hall, London.

[49] Tjøstheim, D., 1986, Some doubly stochastic time series models, J. of Time Series Analysis, 7, $51-72$.

[50] Tong, H., 1990, Nonlinear Time Series: A Dynamical Approach, Oxford University Press.

[51] Tsay, R., 1997, Unit root tests with Threshold Innovations, preprint, University of Chicago.

[52] Weiss, A.,1987, Estimating Nonlinear Dynamic Models Using Least Absolute Error Estimation, Econometric Theory, 7, 46-68.

University of IlLinois at URBANA-Champaign 